\title{
Cuidados paliativos em medicina fetal
}

\author{
Palliative care in fetal medicine
}

\begin{abstract}
Renata Bolibio ${ }^{1,2}$, Roberta Carolina de Almeida Jesus ${ }^{2,3}$, Fernanda Figueiredo de Oliveira ${ }^{2,3}$, Maria Augusta Bento Cicaroni Gibelli, ${ }^{2,4}$, Glaucia Rosana Guerra Benute ${ }^{2,3}$, Ana Lucia Gomes ${ }^{2,3}$, Nathalia Bertolassi Oliveira do Nascimento ${ }^{2,5}$, Tercilia Virginia Aparecida Barbosa ${ }^{2,6}$, Marcelo Zugaib $^{2,3}$, Rossana Pulcineli Vieira Francisco ${ }^{2,3}$, Lisandra Stein Bernardes ${ }^{2,3}$
\end{abstract}

Bolibio R, Jesus RCA, Oliveira FF³, Gibelli MABC, Benute GRG, Gomes AL, Nascimento NBO, Barbosa TVA, Zugaib M, Francisco RPV, Bernardes LS. Cuidados paliativos em medicina fetal / Palliative care in fetal medicine. Rev Med (São Paulo). 2018 mar.-abr:;97(2):208-15.

RESUMO: Malformações fetais acometem cerca de 3\% das gestações, e a possibilidade desse diagnóstico é uma das principais preocupações vivenciadas pelos pais e familiares. Quando é diagnosticado uma malformação que implique em possível mortalidade da criança, é necessário o planejamento do seguimento da gestação, o que abrange diversos aspectos que incluem avaliação minuciosa do prognóstico, organização do cuidado após o parto, e seguimento da família de forma abrangente e integrada. Recentemente os conceitos de cuidados paliativos foram introduzidos na perinatologia com essa finalidade, e o presente artigo tem como objetivo discutir o modelo de cuidado paliativo aplicado no atendimento a gestantes e familiares de fetos com malformação.

Descritores: Cuidados paliativos; Feto/anormalidades; Perinatologia.
ABSTRACT: Fetal malformations affect approximately 3\% of pregnancies, and the possibility of this diagnosis is one of the main concerns of parents and family members. When a malformation that implies potential mortality for the child is diagnosed, the pregnancy's follow-up needs to be planned, including diverse aspects such as thorough evaluation of the prognosis, organization of post-partum care, and follow-up with the family in a broad and integrated manner. Recently, palliative care concepts have been introduced into perinatology for this purpose. This article discusses the application of the palliative care model to the care of pregnant women and family members of fetuses with malformations.

Keywords: Palliative care; Fetus/abnormalities; Perinatology.

1. Divisão de Psicologia do Instituto Central do Hospital das Clínicas da Faculdade de Medicina da Universidade de São Paulo. ORCID: Bolibio R 0000-0002-3948-1767.

2. Grupo de Apoio Integral à gestantes e familiares de fetos com malformação (GAI) da Clínica Obstétrica e Instituto da Criança do Hospital das Clínicas da Faculdade de Medicina da Universidade de São Paulo. ORCID: Jesus RCA - 0000-0002-5022-3398; Oliveira FF - 0000-0002-6278-2525; Benute GRG - 0000-0003-4545-1887.

3. Disciplina de Obstetrícia, Departamento de Obstetrícia e Ginecologia, Faculdade de Medicina da Universidade de São Paulo. ORCID: Francisco RPV - 0000-0002-9981-8069; Bernardes LS - 0000-0003-2367-2849.

4. Departamento de Pediatria do Instituto da Criança Hospital das Clínicas da Faculdade de Medicina da Universidade de São Paulo. ORCID: Gibelli MABC - 0000-0001-9074-7500; Gomes AL - 0000-0001-5083-8360

5. Divisão de Enfermagem do Instituto Central do Hospital das Clínicas da Faculdade de Medicina da Universidade de São Paulo. ORCID: Nascimento NBO - 0000-0002-3281-3896.

6. Divisão de Serviço Social do Instituto Central do Hospital das Clínicas da Faculdade de Medicina da Universidade de São Paulo. ORCID: Barbosa TVA - 0000-0001-7388-2102.

Endereço para correspondência: Lisandra Stein Bernardes. Av. Dr. Enéas de Carvalho Aguiar, 255, 10 andar - Obstetrícia. São Paulo, SP, Brasil. E-mail: lisbernardes@usp.br. 


\section{INTRODUÇÃO E HISTÓRIA}

gestação é um momento, durante o ciclo reprodutivo da mulher, de intensas mudanças corporais, emocionais e sociais. Durante a gestação, o casal e seus familiares desenvolvem expectativas sobre o filho ou criança esperada, entre elas, a expectativa de um filho saudável ${ }^{1}$. No entanto essa expectativa pode não ser correspondida em algumas situações, particularmente quando existe algum diagnóstico que coloca em risco a gestação ou o filho. Malformações fetais acometem cerca de $3 \%$ das gestações, e em aproximadamente 1,2\% das gestações são diagnosticadas malformações fetais extensas ${ }^{2}$, algumas com prognóstico letal ${ }^{3,4}$ o que influencia de forma direta as expectativas do casal.

Independente da gravidade da doença, o diagnóstico de malformação fetal impacta a gestante e seus familiares, sendo recorrente alterações emocionais como ansiedade e preocupações ${ }^{5,6,7,8}$. Dentre os possíveis impactos e sentimentos gerados pela presença de malformação fetal, Andrade ${ }^{9}$ descreve sentimento de tristeza, sofrimento pela possibilidade da perda do filho, sensação de culpa pela malformação, preocupação com a possibilidade do filho sofrer e com a reação de outros filhos diante da descoberta da malformação fetal de alta mortalidade.

No Brasil, há um número grande de malformações fetais diagnosticadas durante o pré-natal e, devido escolha pessoal, diagnóstico tardio ou restrição jurídica, muitas gestantes levam a gestação a termo. É essencial oferecer $\mathrm{o}$ atendimento integral para as gestantes, proporcionando o suporte adequado e nos casos de maior gravidade, a organização do seguimento da gestação e da tomada de decisão ${ }^{10}$.

Na ocasião de um diagnóstico de malformação fetal, indica-se a avaliação detalhada das características da doença fetal, incluindo a avaliação do prognóstico fetal e o planejamento do cuidado e seguimento da gestação. Existem malformações fetais de baixa gravidade, malformações que cursam com alta mortalidade e aquelas na qual pode não haver possibilidade de tratamento curativo e que irão resultar em óbito durante o pré-natal ou após o parto ${ }^{9}$. Nessa situação, a utilização de conceitos de cuidados paliativos já no período antenatal pode propiciar integração do cuidado, possibilidade de preparo para o parto e menor impacto emocional tardio quando há necessidade de seguimento em centro terciário e risco de morte no período perinatal.

Os cuidados paliativos foram inicialmente descritos na população adulta, e reconhecer e cuidar da dor e do sofrimento do paciente e seus familiares faz parte de suas recomendações ${ }^{11}$. A definição utilizada pela Organização Mundial da Saúde conforme descrita em $2002^{12}$ é:

\footnotetext{
"abordagem que promove a qualidade de vida de pacientes e seus familiares, que enfrentam doenças que ameacem a continuidade da vida, através
}

da prevenção e alívio de sofrimento. Requer a identificação precoce, avaliação e tratamento da dor e outros problemas de natureza física, psicossocial e espiritual" (p.26).

Dessa forma, o cuidado paliativo deve ser indicado desde o diagnóstico, e se baseia nos seguintes princípios: 1) promover o alívio da dor e outros sintomas desagradáveis; 2) afirmar a vida e considerar a morte como um processo natural da vida; 3 ) não acelerar nem adiar a morte; 4) integrar os aspectos psicológicos e espirituais no cuidado ao paciente; 5) oferecer um sistema de suporte que possibilite o paciente viver tão ativamente quanto possível, até o momento de sua morte; 6) oferecer sistema de suporte para auxiliar os familiares durante a doença do paciente e a enfrentar o luto; 7) abordagem multidisciplinar para focar as necessidades dos pacientes e seus familiares, incluindo acompanhamento no luto; 8) melhorar a qualidade de vida e influenciar positivamente o curso da doença; 9) deve ser iniciado o mais precocemente possível, juntamente com outras medidas de prolongamento da vida, como quimioterapia e radioterapia e incluir todas as investigações necessárias para melhor compreender e controlar situações clínicas estressantes ${ }^{12}$.

Após o início e estabelecimento do seguimento de adultos e posteriormente de crianças nos moldes preconizados pelos cuidados paliativos, identificou-se a necessidade de sua expansão para os cuidados em perinatologia. Os primeiros registros de modelo de atendimento utilizando o conceito de cuidado paliativo em medicina fetal são da década de 90. Nos Estados Unidos, em 1997, houve a primeira discussão médica sobre a possibilidade de seguimento e planejamento do cuidado com o feto diagnosticado com doença letal no período prénatal, utilizando como referência os valores da família ${ }^{13}$.

A mesma equipe propôs, pela primeira vez em 2001, um modelo de seguimento para famílias que decidissem levar a gestação a termo após o diagnóstico de malformação fetal letal ou de alta mortalidade. A possibilidade de diagnóstico pré-natal acurado e a avaliação de prognóstico eram associadas aos conhecimentos sobre o manejo de luto perinatal e o conceito de Hospice advindo dos cuidados paliativos de adultos, criando a possibilidade de seguimento integrado e centrado na família. Nos anos seguintes a ideia dessa forma de acompanhamento após diagnóstico de malformação fetal se expandiu, e outros países no mundo passaram a propor diferentes formas de atendimento ${ }^{14}$.

\section{Histórico de cuidados paliativos e cuidados paliativos pré-natais no Brasil}

O cuidado paliativo no Brasil iniciou na década de 1980 e atingiu um crescimento significativo a partir do ano $2000^{12}$. Em 2006, o Conselho Federal de Medicina ${ }^{15}$ publicou a resolução $n^{\circ} 1.805 / 2006$, que determina: "É permitido ao médico limitar ou suspender procedimentos 
e tratamentos que prolonguem a vida do doente em fase terminal, de enfermidade grave e incurável, respeitada a vontade da pessoa ou de seu representante legal".

Ainda, segundo o Código de Ética Médica ${ }^{16}$ :

"Nas situações clínicas irreversíveis e terminais, o médico evitará a realização de procedimentos diagnósticos e terapêuticos desnecessários e propiciará aos pacientes sob sua atenção todos os cuidados paliativos apropriados (...) Nos casos de doença incurável e terminal, deve o médico oferecer todos os cuidados paliativos disponíveis sem empreender ações diagnósticas ou terapêuticas inúteis ou obstinadas, levando sempre em consideração a vontade expressa do paciente ou, na sua impossibilidade, a de seu representante legal" (Código de Ética, 2009 - Princípio Fundamental XXII e parágrafo único do artigo 41).

Já em 2006 se iniciaram as discussões sobre cuidado paliativo no período neonatal no Brasil. Bueno ${ }^{17}$ reforça a importância do cuidado paliativo no período neonatal, incluindo a família no processo de assistência ao recém-nascido em fim de vida e discutindo objetivos no cuidado.

No ano seguinte, autores ${ }^{18}$ relatam que os cuidados paliativos neonatais são indicados nos casos em que "não há tratamento disponível que proporcione alteração substancial na progressão do quadro clínico para a morte". Afirmam que quando o diagnóstico é feito precocemente, o período gestacional é a oportunidade para o planejamento do período pós-natal ${ }^{18}$.

No entanto, até o ano de 2017, não havia no Brasil descrição de modelo de seguimento em cuidados paliativos se iniciando no período pré-natal. A utilização de conceitos de cuidado paliativo pré-natal no atendimento de fetos com malformação de alta mortalidade foi descrita nesse ano em nosso país ("Grupo de atendimento integral às gestantes e familiares de fetos com malformação: utilização de conceitos de cuidados paliativos no atendimento em medicina fetal"). No modelo descrito, que foi implementado com sucesso na Clínica Obstétrica do Hospital das Clínicas da Universidade de São Paulo, possibilita-se o seguimento integrado das famílias após o diagnóstico de malformação fetal de forma a prepará-las para o parto e período após o parto, permitir escolhas conscientes em relação ao cuidado com o filho e incluir os valores individuais das famílias no preparo do seguimento ${ }^{9}$.

Fetos elegíveis para seguimento em modelo de cuidados paliativos

Os fetos elegíveis para seguimento em cuidados paliativos são aqueles para os quais há diagnóstico de doenças que limitam a vida, o que inclui a maioria das malformações fetais maiores (malformações que necessitam cirurgia ou que levam à restrição no tempo ou qualidade de vida).As diferenças culturais entre os diversos países fazem com que a seleção de famílias elegíveis para seguimento em cuidados paliativos pré-natais seja individualizada, considerando-se valores inerentes à cada país e sociedade.

Historicamente, Hoeldtke e Calhoun ${ }^{14}$ sugerem que, em países nos quais o aborto terapêutico é permitido, juntamente com a discussão da possibilidade da realização do aborto seja proposta a opção de dar continuidade a gestação e realizar o seguimento em cuidados paliativos pré-natal a todas as famílias candidatas. Ressaltam que, nessa discussão, o seguimento da gestação não deve ser apresentado à família como uma atitude passiva.O seguimento em cuidados paliativos pré-natal proporciona formas de cuidado com o feto e o recém-nascido discutidas ativamente em conjunto, equipe e família, propiciando a aproximação e criação de vínculo com o filho gerado, ainda que o mesmo não sobreviva por muito tempo.

Leuthner ${ }^{19}$ debate sobre a aplicação dos cuidados paliativos no período pré-natal e na sala de parto, incluindo doenças que possam ameaçar a continuidade da vida, mas para as quais deve ser prestado tratamento inicial e avaliação de resposta neonatal. De acordo com o autor, o processo de decisão do tipo de cuidado a ser oferecido implica no conhecimento de três fatores básicos: o diagnóstico; o prognóstico e o significado do prognóstico para os pais.

$\mathrm{Na}$ Inglaterra, a British Association of Perinatal Medicine ${ }^{20}$ apresenta como indicações de seguimento fetos com diagnóstico de doenças não compatíveis com sobrevida a longo prazo e diagnóstico de condições que impliquem em risco de óbito ou mortalidade significativa.

No Brasil, no modelo descrito por Andrade ${ }^{9}$, todos os fetos que têm malformações candidatas à seguimento em centro terciário são elegíveis para acompanhamento. Como há grande volume de famílias atendidas anualmente, as famílias de fetos com mortalidade estimada inferior 50\% são acompanhadas em grupos de discussão, e aquelas que apresentam fetos com doença de maior mortalidade (mortalidade estimada acima de 50\%) recebem atendimento individual.

Em conclusão, em nossa sociedade, a partir do momento em que se diagnostica alguma malformação que coloque em risco a vida da criança, a família é elegível para seguimento em cuidados paliativos perinatais, possibilitando acompanhamento integral desde o momento do diagnóstico até o período após o parto.

\section{Avaliação de prognóstico de fetos seguidos em cuidados paliativos no período pré-natal}

A avaliação de prognóstico fetal é essencial para a equipe e para o aconselhamento das famílias, e um dos primeiros passos no seguimento. É a partir dessa avaliação que se iniciam as discussões sobre os possíveis desfechos e sobre a conduta que será tomada. Ainda, é a partir da avaliação de prognóstico que se inicia o planejamento da comunicação com a gestante e seus familiares. 
Nessa avaliação, conforme sugerido por Leuthner ${ }^{19}$, pode se pensar em doenças letais, para as quais o desfecho é o óbito em pouco tempo de vida, como no caso da anencefalia $^{21}$, e doenças cujo prognóstico é incerto e a mortalidade é alta, mas para as quais há possibilidade de tratamento que modifique o curso da doença, como a hérnia diafragmática congênita grave ${ }^{22}$.

É importante ressaltar-se que a classificação da doença fetal como letal não implica óbito imediatamente após o parto. Há, na maior parte das doenças, possibilidade de óbito em diversos períodos da gestação e pós-parto (óbito intra-útero, intra-parto, nas primeira24horas, ou mesmo alguns dias ou meses após o parto $)^{9}$.

Andrade $^{9}$ apresenta o desfecho considerando fetos com prognóstico indeterminado e letal e descreve a ampla possibilidade de tempo de óbito, o que deve ser extensivamente discutido com os pais se estes assim o desejarem.

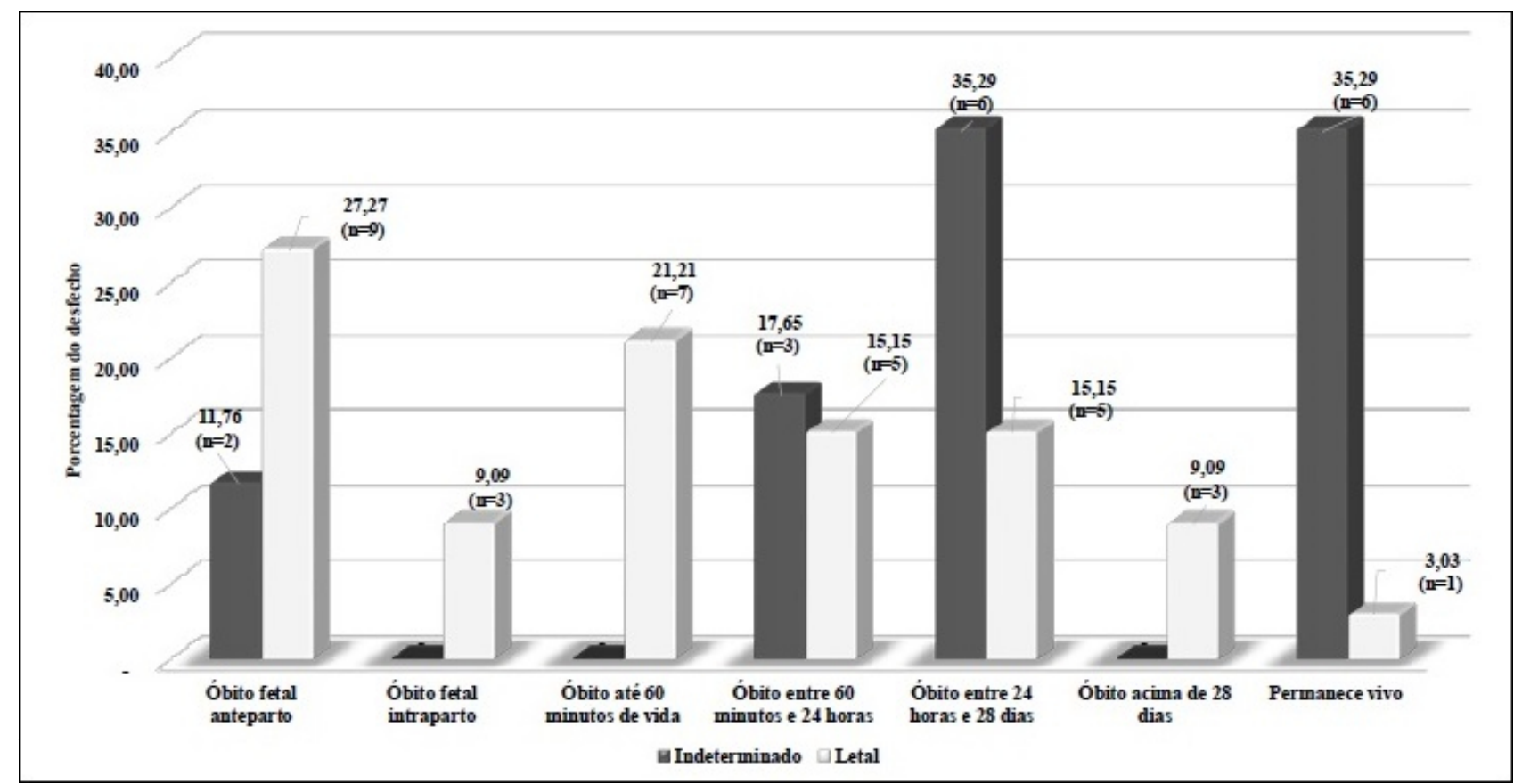

Figura 1. Desfecho dos fetos das gestantes seguidas no GAI de maio de 2015 a setembro de 2016 - HCFMUSP 2017

Além do momento do óbito, é importante que seja discutido que o seguimento em modelo de cuidado paliativos parece não modificar o desfecho das crianças.

Em um estudo retrospectivo avaliando os resultados pós-natais de uma população de 49 recém-nascidos com diagnostico pré-natal de condições que limitam a vida e cujos pais foram encaminhados para aconselhamento no pré-natal com a equipe, Parravicine e Lorenz ${ }^{23}$ observaram que todos os recém-nascidos morreram com idade média similar de 2 dias (tanto os 28 que foram assistidos com medidas de conforto com os 17 que receberam cuidados intensivos). Assim, os autores concluíram que os cuidados intensivos não previnem a morte de bebês afetados por condições que limitam a vida.

Kukora et al. ${ }^{24}$ realizaram um estudo comparando gestantes que participaram de uma consulta de cuidado paliativo pré-natal e gestantes que não participaram, com o objetivo de descrever o seguimento de fetos com prognostico de doença letal ou de alta mortalidade e averiguar se a consulta modificou a sobrevida a longo prazo dos fetos. Os autores observaram que, embora o prognóstico dos fetos das gestantes que realizaram a consulta fosse mais grave, não houve diferença da mortalidade a longo prazo: apresentando $21 \%$ de morte fetal, $67 \%$ de morte hospitalar e $12 \%$ de sobrevivência para alta. Ao mesmo tempo, observaram que a frequência de famílias que optaram pela não utilização de suporte artificial de vida foi significativamente maior quando as gestantes realizaram uma consulta de cuidado paliativo e essas famílias foram mais propensos a escolher cuidados de conforto e maior contato com o filho sem intermédio de aparelhos.

\section{Seguimento em cuidados paliativos perinatais durante o pré-natal}

Durante as conferências familiares realizadas no período pré-natal, a equipe de saúde tem a possibilidade de avaliar as necessidades individuais de cada família e identificar seus valores. Para tanto, é fundamental reunir habilidades de profissionais de diferentes áreas de forma que cada um reconheça suas capacidades e limites de atuação ${ }^{11}$.

Hoeldtke e Calhoun ${ }^{14}$ sugerem os seguintes profissionais como essenciais na construção da equipe: um obstetra, um enfermeiro e um assistente social. Além desses 
recomenda-se inclusão de outros membros, incluindo membros da neonatologia, psicologia e capelania segundo necessidades específicas das famílias e possibilidades de cada instituição.

O seguimento deve atender as demandas das famílias, sendo necessário flexibilidade da equipe. É essencial que os membros da equipe assegurem aos familiares que estarão presentes e darão o suporte necessário durante o seguimento, identificando as possíveis causas de sofrimento, inclusive durante o parto e a morte do recém-nascido caso esta ocorra. Sugerem que os pais sejam guiados na interação com outros filhos, familiares e amigos quando necessário, e que o feto seja acolhido como um indivíduo, por toda a equipe, permitindo a construção de lembranças para a gestação ${ }^{11}$.

Os autores Hoeldtke e Calhoun ${ }^{14}$ propõem que, durante o pré-natal, seja discutido com os pais o cuidado que será dado ao recém-nascido se este nascer vivo.

Leuthner ${ }^{25}$ retoma o planejamento do seguimento da gestação proposto por Hoeldtke e Calhoun ${ }^{14}$ e inclui a organização do plano de cuidado com o recém-nascido no planejamento para o parto, além de sugerir auxílio prático na organização dos procedimentos e rituais após o óbito quando houver demanda dos pais.

Howard ${ }^{26}$ propõe que nos casos de diagnóstico de malformação fetal maior, o cuidado deve ser centrado na família. Sendo a gravidez um período de transição biológica e psicológica na vida da mulher, o diagnóstico de malformação fetal faz com que este período seja emocionalmente devastador e com pouco suporte social. Para o autor, o seguimento tem como princípio determinar as preferências e necessidades individuais de cada membro da família, com o objetivo de proporcionar um guia antecipatório dos possíveis desfechos, quando a família desejar.

Bhatia $^{27}$ afirma que o durante o atendimento de pré-natal, deve ser discutida a mortalidade estimada de cada doença, as chances de sequela e o tipo de tratamento para casa caso, permitindo que a tomada de decisão seja conduzida pelo interesse do feto, em conjunto com a família.

No modelo brasileiro descrito, os atendimentos ambulatoriais do GAI (Grupo de Apoio Integral à Gestante e Familiares de Fetos com Malformação) são realizados por dois membros da equipe, preferencialmente de profissões diferentes, no modelo de conferência familiar. As gestantes são incentivadas a comparecer às consultas pré-agendadas com os familiares que desejarem, sendo destacado a importância da presença do genitor do feto ou parceiro. Nesse modelo, em um dos primeiros passos após o encaminhamento, a equipe discute o prognóstico em reunião semanal do grupo com o intuito de, a partir da literatura existente, discutir a conduta médica para a gestante, feto e neonato e homogeneizar as informações de todos os membros da equipe ${ }^{9}$.
Em seguida iniciam-se as conferências familiares com o intuito conhecer a família e identificar seus valores, bem como percepções sobre a gestação, o parto e os cuidados a serem realizados. Nestas conferências, "são discutidas as consequências das diversas vias de parto e formas de monitorização fetal e de suporte neonatal possiveis, bem como as consequências dos objetivos de cuidado pensados com a família"9.

A partir do conhecimento profundo da família, e do estabelecimento das condutas médicas propostas pela equipe, começa-se a construção do processo de decisão conjunta acerca do cuidado ao longo de todo o seguimento, o que inclui a construção do plano de parto.

\section{Construção de plano de parto}

O planejamento do parto configura um dos passos fundamentais no seguimento das famílias. Segundo English e Hessler ${ }^{28}$, o plano de cuidado está incluído no plano de parto, e é construído de forma a permitir aos pais participação nas escolhas em relação ao filho, considerando o que avaliam como conforto, o período em que os membros da família permanecerão juntos e a dignidade do breve momento que esses pais terão com o filho em casos de doença letal. Inclui orientação sobre o que esperar no momento do parto, como será o ambiente, o local de atendimento, planejamento de registros como fotos e outras lembranças, que, além de importantes nas memórias, podem ser importantes no processo de luto caso a criança venha a óbito.

Ainda, quando há fetos com diagnóstico de doenças cuja sobrevida esperada é curta, a partir de uma investigação sobre o que os pais consideram importante, busca-se estabelecer como os pais irão usufruir dos últimos minutos, horas ou dias do recém-nascido.Caso o recémnascido nasça com vida, pode ser permitido e sinalizado no plano de parto que seja fosse colocado no colo dos pais, quando esses o desejarem, e que os pais fiquem com o filho o tempo desejado, inclusive assegurando que o cuidado seja prestado no colo quando possível ${ }^{14}$.

Em determinados casos, se for possível e de interesse da família podem ser discutidos os procedimentos a serem realizados após o óbito, podendo ser entregue uma cartilha de orientação após o óbito?.

\section{Momento do parto}

O momento do parto é um dos momentos mais estressantes e angustiantes para a gestante e os familiares quando existe o diagnóstico de malformação fetal maior ${ }^{10}$.

Suassuna $^{29}$ afirma que o filho idealizado é perfeito. Diferente do filho idealizado, o filho com malformação é o filho real. Este filho não mais preenche o papel que lhe era destinado no cenário familiar. Essa grande frustração pode gerar sérias rupturas na relação entre pais e bebês, 
impactos significativos no processo de vínculo de ambos os genitores, sentimentos de culpa, agressividade e tristeza.

O corte concreto do cordão umbilical interromperá a oxigenação placentária do feto, dando lugar à manifestação de sintomas de doenças cardíacas, pulmonares e cromossômicas que, muitas vezes, pouco se manifestavam na vida intra-uterina. Além disso, quando há doença de curta sobrevida, o momento do parto é o momento no qual pode iniciar-se o fim da vida. Por esse motivo, o preparo da família para esse momento faz-se de extrema importância 9 .

É importante que a equipe de plantão, quando o parto é realizado por plantão, tenha conhecimento dos aspectos discutidos e acordados com a família durante o seguimento pré-natal. Isso pode ser feito a partir dos registros em prontuário e no cartão de pré-natal, e pelo próprio plano de parto. Além disso, a equipe médica de plantão deve conversar com a gestante no momento da sua admissão para verificar se há alguma dúvida ou mudança em relação a conduta definida previamente nas conferências ${ }^{9}$.

\section{Seguimento pós-natal}

O seguimento das famílias após o parto é de suma importância, seja para as aquelas cujo filho morreu e que passarão prelo processo de luto, seja para aquelas que necessitam suporte para acompanhá-lo pelo processo de cirurgias e estadia em unidade de terapia intensiva inerente à maior parte das malformações maiores tratáveis. Validando o princípio de oferecer suporte para auxiliar os familiares durante a doença do paciente e a enfrentar o luto, o "Manual de cuidados paliativos"12 afirma que a assistência a família do doente requer do grupo a capacidade de manejar situações de conflito, estimular o doente e família a pensarem e falarem livremente sobre sua a vivência, legitimar o sofrimento e contribuir para a elaboração das experiências de adoecimento, processo de morte e luto.É importante ainda que haja suporte ao luto disponível por até 12 meses após o óbito, para que se previna e se identifique características de luto complicado.

Além disso, é importante que seja observado de que forma o seguimento pré-natal influenciou o parto, e como a família se sentiu nesse processo?.

Em suma, considerando-se os passos no cuidado com as famílias após diagnóstico de malformação fetal e a análise de 50 famílias acompanhadas no modelo proposto, Andrade ${ }^{9}$ propôs modelo teórico para seguimento e tomada de decisões em cuidados paliativos após diagnóstico de malformação fetal.

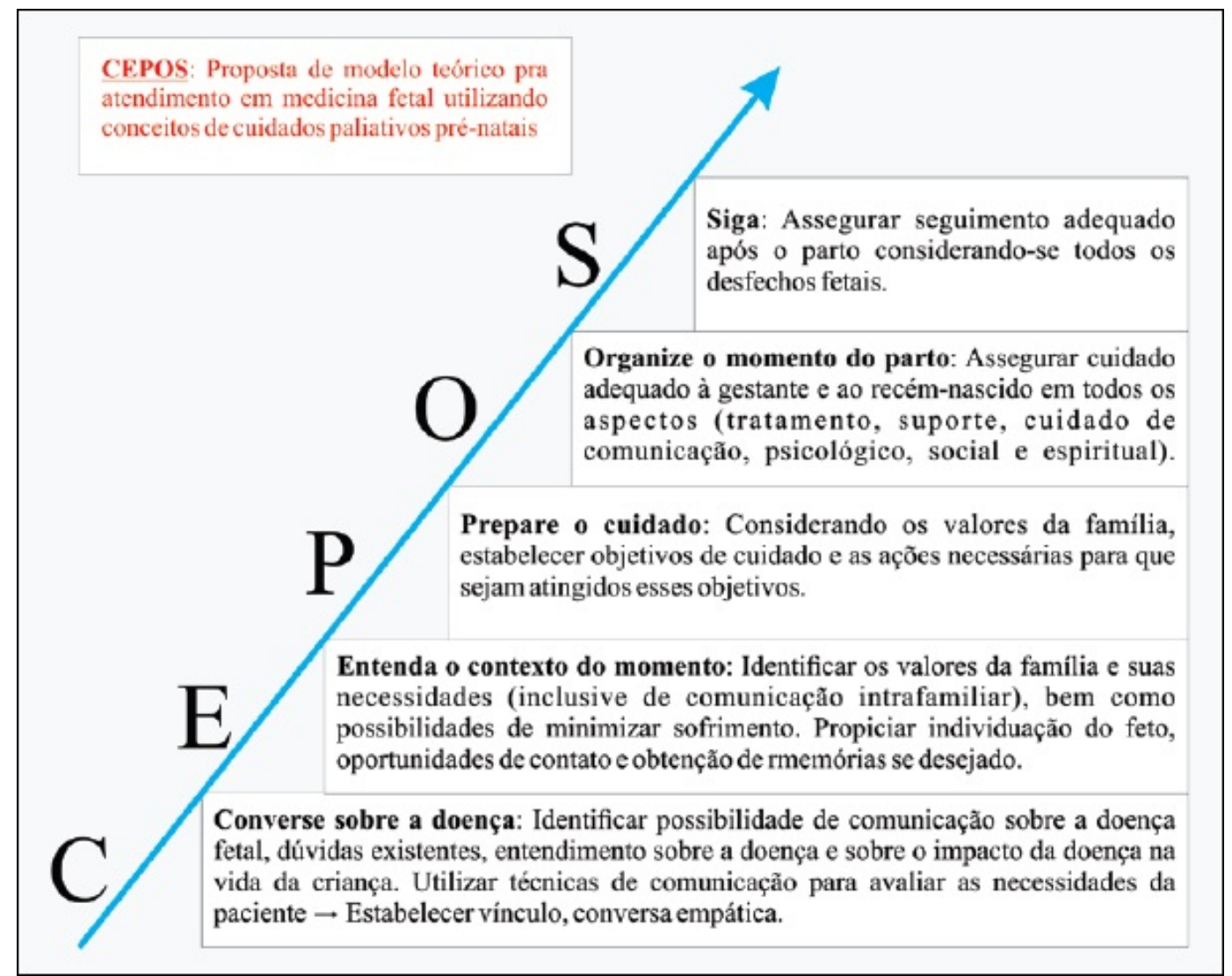

Reprodução autorizada por Lisandra Stein Bernardes Campi de Andrade.

Figura 2. Modelo teórico para seguimento e tomada de decisões utilizando conceitos de cuidados paliativos no seguimento em medicina fetal 


\section{CONCLUSÃO}

Com o objetivo de realizar o seguimento integral do feto diagnosticado com malformação, da gestante e de seus familiares, a utilização do modelo de cuidado paliativo

\section{REFERÊNCIAS}

1. Machado MEC. Casais que recebem um diagnóstico de malformação fetal no pré-natal: uma reflexão sobre a atuação do psicólogo hospitalar. Rev SBPH. 2012;15(2):8595. Disponível em: http://pepsic.bvsalud.org/scielo. php?script=sci_arttext\&pid=S1516-08582012000200007.

2. European Surveillance of Congenital Anomalies (EUROCAT). EUROCAT guide 1.4: instruction for the registration of congenital anomalies. EUROCAT Central Registry, University of Ulster; 2013 [cited 2017 Oct 09]. Available from: http://www.eurocat-network.eu/content/ Full\%20Guide\%201\%204\%20v5\%2020_Dec2016.pdf.

3. Romosan G, Henriksson E, Rylander A, Valentin L. Diagnostic performance of outine ultrasound screening for fetal abnormalities in an selected Swedish population in 2000-2005. Ultrasound Obstet Gynecol. 2009;34:526-33. doi: 10.1002/uog.6446.

4. Farraposo S, Montenegro N, Matias A. Evaluation of the role of first-trimester obstetric ultrasound in the detection of major anomalies: a systematic review. J Perinat Med. 2014;42:141-9. doi: 10.1515/jpm-2013-0130.

5. Shulth W, Karck U, Wilhelm C, Reisch S. Parents' needs after ultrasound diagnosis of a fetal malformation: an empirical deficit analysis. Ultrasound Obstet Gynecol. 1994;4:124-9. doi: 10.1046/j.1469-0705.1994.04020124.x.

6. Leithner K, Maar A, Fischer-Kern M, Hilger E, LöfflerStastka H, Ponocny-Seliger E. Affective state of women following a prenatal diagnosis: predictors of a negative psychological outcome. Ultrasound Obstet Gynecol. 2004;23(3):240-6. doi: 10.1002/uog.978.

7. Debost-Legrand A, Laurichesse-Delmas H, Francannet C, Perthus I, Lémery D, Gallot D, Vendittelli F. False positive morphologic diagnoses at the anomaly scan: marginal or real problem, a population-based cohort study. BMC Pregnancy Childbirth. 2014;14:112. doi: 10.1186/1471-2393-14-112.

8. Titapant V, Chuenwattana P. Psychological effects of fetal diagnoses of non-lethal congenital anomalies on the experience of pregnant women during the remainder of their pregnancy. J Obstet Gynaecol Res. 2015;41(1):77-83. doi: 10.1111/jog. 12504.

9. Andrade LSBC. Grupo de apoio integral às gestantes e familiares de fetos com malformação: utilização de conceitos de cuidados paliativos no atendimento em medicina fetal [Tese Livre Docência]. São Paulo: Faculdade de Medicina da Universidade de São Paulo; 2017.

10. Benute GR, Nomura RM, Liao AW, Bizot ML, Lucia MCS, Zugaib M. Feelings of women regarding end-of-life decision making after ultrasound diagnosis of a lethal fetal malformation. Midwifery. 2012;28(4):472-5. doi: 10.1016/j. midw.2011.06.011. na organização do atendimento possibilita planejamento conjunto da gestação, do parto e do pós-parto, adequando o seguimento aos valores e demandas de cada família e permitindo tomada compartilhada de decisões.

11. Hermes HR, Lamarca ICA. Cuidados paliativos: uma abordagem a partir das categorias profissionais de saúde. Ciênc Saúde Coletiva. 2013;18(9):2577-88. http://dx.doi. org/10.1590/S1413-81232013000900012.

12. Carvalho RT, Parsons HA, organizadores. Manual de cuidados paliativos ANCP. 2a ed. ampl. atual. São Paulo: ANCP; 2012 [citado 09 out. 2017]. Disponível em: http://www.paliativo. org. br/noticias/ ztag/manual-de-cuidados-paliativos-ancp/.

13. Calhoun BC, Reitman JS, Hoeldtke NJ. Perinatal hospice: a response top artial birth abortion for infants with congenital defects. Issues Law Med. 1997;13(2):125-43.

14. Hoeldtke NJ, Calhoun BC. Perinatal hospice. Am J Obstet Gynecol. 2001;185(3):525-9. doi: 10.1067/mob.2001.116093

15. Conselho Federal de Medicina (CFM). Resolução CFM no 1.805/2006. Brasília: CFM; 2009 [citado 15 out. 2017]. Disponível em: http://www.portalmedico.org.br/resolucoes/ cfm/2006/1805_2006.htm.

16. Conselho Federal de Medicina (CFM). Código de ética médica. Resolução CFM n ${ }^{\circ} 1.931$, de 19 de setembro de 2009 [citado 15 out. 2017]. Brasília: CFM; 2009. Disponível em: https://portal.cfm.org.br/images/stories/biblioteca/ codigo $\% 20$ de $\% 20$ etica $\% 20$ medica.pdf.

17. Bueno M, Bussotti EA, Sakita NK, Barbosa SMM. Reflexões sobre cuidados paliativos no período neonatal. Prática Hospitalar. 2007;50:87-90.

18. Barbosa SMM, Souza JL, Bueno M, Sakita NK, Buussotti EA. Período neonatal. In: Oliveira, coordenador. Cuidado paliativo. São Paulo: CFM; 2008. p.139-52.

19. Leuthner SR. Fetal palliative care [review]. Clin Perinatol. 2004;31(3):649-65. doi: 10.1016/j.clp.2004.04.018.

20. British Association of Perinatal Medicine Working Group Report. Palliative care (supportive and end of life care) A framework for clinical practice in perinatal medicine. 2010. Available from: https://www.bapm.org/sites/default/files/ files/Palliative_care_final_version_\%20Aug10.pdf.

21. Jaquier M, Klein A, Boltshauser E. Spontaneous pregnancy outcome after prenatal diagnosis of anencephaly. BJOG. 2006;113(8):951-3.

22. Shan W, Wu Y, Huang G, Zeng L, Miao, Yuan, Huang L, Xiang B, Jiang X. Foetal endoscopic tracheal occlusion for severe congenital diaphragmatic hérnia - a systemic review and meta-analysis of randomized controlled trials. J Pak Med Assoc. 2014;64(6):686-9. Available from: http://jpma.org.pk/ full_article_text.php?article_id $=6652$.

23. Parravicini E, Lorenz JM. Neonatal outcomes of fetuses diagnosed with life-limiting conditions when individualized comfort measures are proposed. J Perinatol. 2014;34(6):4837. doi: $10.1038 /$ jp.2014.40. 
24. Kukora S, Gollehon N, Laventhal N. Antenatal palliative care consultation: implications for decision-makingand perinatal outcomes in a single-centre experience. Arch Dis Child Fetal Neonatal Ed. 2016;102(1):F12-16. doi: 10.1136/ archdischild-2016-311027.

25. Leuthner SR. Palliative care of the infant with lethal anomalies. Pediatr Clin North Am. 2004;51(3):747-59. https://doi. org/10.1016/j.pcl.2004.01.006.

26. Howard ED. Family-centered care in the context of fetal abnormality. J Perinat Neonatal Nurs. 2006;20(3):237-42.
27. Bhatia J. Palliative care in the fetus and newborn. J Perinatol. 2006;26(1):S24-6. doi: 10.1038/sj.jp.7211468

28. English NK1, Hessler KL. Prenatal birth planning for families of the imperiled newborn. J Obstet Gynecol Neonatal Nurs. 2013;42(3):390-9. https://doi.org/10.1111/1552-6909.12031

29. Suassuna AMV. A influência do diagnóstico pré-natal na formação de possíveis psicopatologias do laço mãe-bebê [dissertação]. São Paulo: Universidade Presbiteriana Mackenzie; 2008. 